\title{
NUMERICAL SOLUTION OF THE MULTI-TERM VARIABLE-ORDER SPACE FRACTIONAL NONLINEAR PARTIAL DIFFERENTIAL EQUATIONS
}

\author{
H. ÇERDIK YASLAN \\ Received 07 October, 2020
}

\begin{abstract}
A numerical approach for solving the multi-term variable-order space fractional nonlinear partial differential equations is proposed. The fractional derivatives are described in the Caputo sense. The numerical approach is based on generalized Laguerre polynomials and finite difference method. The proposed scheme transforms the main problem to a system of nonlinear algebraic equations. The nonlinear system is solved by using Newton's method. The validity and the applicability of the proposed technique are shown by numerical examples.
\end{abstract}

2010 Mathematics Subject Classification: 35G31; 35R11; 65M70

Keywords: nonlinear multi-term fractional partial differential equation, the variable-order Caputo fractional derivative, finite difference method, Newton's method, generalized Laguerre polynomials

\section{INTRODUCTION}

Fractional differential equations play a significant role in various fields of science and engineering such as fluid mechanics, mechanics of materials, biology, plasma physics, finance, chemistry, image processing (see, for example, [1-4,14,18-20,26]). Variable-order fractional calculus is an extension of the constant-order fractional calculus. In variable-order fractional calculus, the orders of fractional integrals and derivatives are functions in time and space variables. Variable-order fractional derivatives have important applications in various fields of science and engineering such as physics, mechanics [6], viscoelasticity oscillators [21], signal processing [24] and optimization control [27]. Since variable-order fractional nonlinear partial differential equations are much more complicated than other fractional differential equations and have an important place in the application areas, researchers have recently started to work on the solution of such equations.

The solutions of the nonlinear variable-order time fractional partial differential equations have been investigated in $[8,11]$. An optimization method based on the 
generalized polynomials has been proposed for solving the variable-order time fractional Burgers'equation [8]. Stable three-level explicit scheme for a class of nonlinear variable-order time fractional partial differential equations has been studied [15]. Solving two-dimensional variable-order time fractional optimal control problems, a numerical approach has been introduced based on generalized polynomials and the Lagrange multipliers method [16]. Variable-order time fractional nonlinear cable equation and Klein Gordon equation have been solved by using the Chebyshev cardinal operational matrix method and nonstandard finite difference method, respectively $[12,25]$. The moving least squares meshless approach has been applied to the two dimensional variable-order time fractional nonlinear diffusion-wave equation [22]. Variable-order time fractional nonlinear partial differential equations have been solved by using collocation method based on the Legendre - Laguerre polynomials and an optimization method based on the generalized shifted Chebyshev polynomials, respectively $[7,9]$. The solution of the nonlinear variable-order time fractional two-dimensional Klein-Gordon equation has obtained by using a semidiscrete method based on the 2D Legendre wavelets [11]. Variable-order space-time fractional nonlinear diffusion-wave equation has been solved based on the Chebyshev cardinal functions coupling with the tau and collocation methods [10].

In this paper, we consider multi-term nonlinear variable-order space fractional partial differential equation of the form:

$$
\frac{\partial u(x, t)}{\partial t}=\sum_{k=1}^{r} h_{k}(x, t) .{ }^{C} D_{x}^{\alpha_{k}(x, t)} u(x, t)+N(u(x, t)), 0<x<L, 0<t \leq T,
$$

with the initial condition

$$
u(x, 0)=f(x), 0<x<L
$$

and the boundary conditions:

$$
\begin{array}{r}
u\left(b_{j}, t\right)=d_{j}(t), b_{0}=0<b_{1}<\ldots<b_{n_{r}-2}<b_{n_{r}-1}=L, \\
j=0,1, \ldots, n_{r}-1,0<t \leq T,
\end{array}
$$

where $x$ is a space variable, $t$ is a time variable; the parameters $\alpha_{k}(x, t), k=1, \ldots, r$, refer to the order of the Caputo variable-order fractional derivative with respect to the space variable. $0<\alpha_{1}(x, t)<\ldots<\alpha_{r}(x, t), n_{i}-1<\alpha_{i}(x, t) \leq n_{i}, n_{i} \in \mathbb{N}, i=1,2, \ldots, r$, $h_{k}(x), k=1,2, \ldots, r, f(x)$ and $d_{j}(t), j=0,1, \ldots, n_{r}-1$ are known continuous functions. $N(u(x, t))$ represents the nonlinear term.

In the present paper, we obtain an approximate solution of the initial and boundary value problem (1.1)-(1.3) in terms of the Laguerre polynomials. Firstly, unknown function is written in terms of the Laguerre polynomials. Substituting Laguerre polynomial solution into equation (1.1), system of ordinary differential equations for appropriate collocation points is obtained. The finite difference method and Newton's method are applied to the obtained system, respectively. This paper is structured as follows: in Section 2, some basic preliminaries of variable-order Caputo fractional 
derivative and Laguerre polynomials are presented. Spectral method for solving the initial and boundary value problem (1.1)-(1.3) is presented in Section 3. The efficiency and validity of the proposed method is demonstrated by some numerical examples in Section 4. Finally, conclusion is given in Section 5.

\section{PRELIMINARIES AND NOTATIONS}

\subsection{The Variable-Order Caputo Fractional Derivative}

Definition 1. The Caputo variable-order fractional differential operator is given by (see, for example, $[5,23])$

$$
{ }^{C} D_{x}^{\alpha(x, t)} u(x, t)=\frac{1}{\Gamma(n-\alpha(x, t))} \int_{0}^{t}(t-\tau)^{n-\alpha(x, t)-1} \frac{\partial^{n} u(x, \tau)}{\partial \tau^{n}} d \tau .
$$

where $n-1<\alpha(x, t)<n$ and $\Gamma($.$) is a Gamma function.$

Using the above definition, the fractional derivative of a polynomial $x^{m}, m \in \mathbb{N}$ can be obtained as follows:

$$
{ }^{C} D_{x}^{\alpha(x, t)} x^{m}= \begin{cases}\frac{\Gamma(m+1)}{\Gamma(m-\alpha(x, t)+1)} x^{m-\alpha(x, t)}, & m \geq n \\ 0, & m<n .\end{cases}
$$

\subsection{Some Properties of the Generalized Laguerre Polynomials}

The generalized Laguerre polynomials $L_{n}^{a}(x),(a>-1)$ are orthogonal polynomials of degree $n$ in $x$ defined on the interval the $(0, \infty)$. The orthogonality relation is given as follows [13]:

$$
<L_{n}^{a}(x), L_{m}^{a}(x)>=\frac{1}{\Gamma(a+1)} \int_{0}^{\infty} x^{a} e^{-x} L_{n}^{a}(x) L_{m}^{a}(x) d x=\left(\begin{array}{c}
n+a \\
n
\end{array}\right) \delta_{n m} .
$$

The polynomials $L_{n}^{a}(x)$ may be generated by using the recurrence relations

$$
L_{n+1}^{a}(x)=\frac{1}{n+1}\left[(2 n+a+1-x) L_{n}^{a}(x)-(n+a) L_{n-1}^{a}(x)\right], \quad n=1,2,3, \ldots
$$

with $L_{0}^{a}(x)(x)=1, L_{1}^{a}(x)(x)=a+1-x$.

The analytical form of the generalized Laguerre polynomials $L_{n}^{a}(x)$ is given by

$$
L_{n}^{a}(x)=\sum_{k=0}^{n}(-1)^{k} \frac{\Gamma(n+a+1) x^{k}}{(n-k) ! k ! \Gamma(k+a+1)} .
$$

The function $y(x)$ which belongs to the space of square integrable function in $[0, \infty)$ may be expressed in terms of the generalized Laguerre polynomials as

$$
y(x)=\sum_{i=0}^{\infty} c_{i} L_{i}^{a}(x) .
$$




$$
c_{i}=\frac{\Gamma(i+1)}{\Gamma(i+a+1)} \int_{0}^{\infty} y(x) x^{a} e^{-x} L_{i}^{a}(x) d x .
$$

Consider only the first $(m+1)-$ terms of the generalized Laguerre polynomials, so we can write

$$
y_{m}(x)=\sum_{i=0}^{m} c_{i} L_{i}^{a}(x)
$$

Theorem 1. Let $y_{m}(x)$ be approximated function in terms of the generalized Laguerre polynomials $L_{i}^{a}(x)$ given by equation (2.4), then we have

$$
{ }^{C} D_{x}^{\alpha(x, t)} y_{m}(x)=\sum_{i=n}^{m} \sum_{k=n}^{i} c_{i} A_{i, k}^{\alpha} x^{k-\alpha(x, t)}, \quad n-1<\alpha(x, t) \leq n,
$$

where

$$
A_{i, k}^{\alpha}=(-1)^{k} \frac{\Gamma(i+a+1)}{\Gamma(k+1-\alpha) \Gamma(i-k+1) \Gamma(a+k+1)} .
$$

\section{NUMERICAL SCHEME}

In this section, we apply Laguerre collocation method to the problem (1.1)-(1.3). In the Laguerre collocation method, the unknown function is written as a finite series expansion via generalized Laguerre polynomials. Then, the finite difference method is applied to the obtained system of ordinary differential equation. Finally, the nonlinear system is solved by Newton's method and the unknown coefficients of the finite series are obtained.

Let $u_{m}(x, t)$ be the truncated Laguerre approximation of a function $u(x, t)$ as follows:

$$
u_{m}(x, t)=\sum_{i=0}^{m} c_{i}(t) L_{i}^{a}(x) .
$$

Using equations (1.1) and (2.5), we obtain

$$
\sum_{i=0}^{m} \frac{d c_{i}(t)}{d t} L_{i}^{a}(x)=\sum_{j=1}^{r} h_{j}(x, t) \sum_{i=n_{j}}^{m} \sum_{k=n_{j}}^{i} c_{i}(t) A_{i, k}^{\alpha_{j}} x^{k-\alpha_{j}(x, t)}+N\left(\sum_{i=0}^{m} c_{i}(t) L_{i}^{a}(x)\right) .
$$

Now, we collocate (3.2) at $m-n_{r}+1$ points $x_{p}$ as follows:

$$
\sum_{i=0}^{m} \frac{d c_{i}(t)}{d t} L_{i}^{a}\left(x_{p}\right)=\sum_{j=1}^{r} h_{j}\left(x_{p}, t\right) \sum_{i=n_{j}}^{m} \sum_{k=n_{j}}^{i} c_{i}(t) A_{i, k}^{\alpha_{j}} x_{p}^{k-\alpha_{j}\left(x_{p}, t\right)}+N\left(\sum_{i=0}^{m} c_{i}(t) L_{i}^{a}\left(x_{p}\right)\right),
$$

where collocation points $x_{p}$ are the roots of shifted Chebyshev polynomials of the second kinds $U_{m-1}^{*}(x)$.

For a positive integer $N, \Delta t=\frac{T}{N}$ denotes the step size of time variable $t$. So we define $t_{q}=q \Delta t, 0 \leq t_{q} \leq T$, in which $q=0,1, \ldots, N$. And we introduce the 
following notations: $u\left(b_{j}, t_{q}\right)=d_{j}^{q}, j=0,1, \ldots, n_{r}-1, c_{i}\left(t_{q}\right)=c_{i}^{q}, i=0,1, \ldots, m$, $V^{q}=\left(c_{0}^{q}, c_{1}^{q}, \ldots, c_{m}^{q}\right)^{*}$ and $*$ is the sign of transposition of the matrix.

Substituting equation (3.1) into the boundary condition (1.3), we have

$$
u_{m}\left(b_{j}, t\right)=\sum_{i=0}^{m} c_{i}(t) L_{i}^{a}\left(b_{j}\right)=d_{j}(t), j=0,1, . ., n_{r}-1 .
$$

By using equations (2.3) and (3.1) for initial condition (1.2), we can compute the unknown constants $c_{i}\left(t_{0}\right), i=0,1, \ldots, m$, i.e. $V^{0}$.

Applying the finite difference method to the system (3.3), we have

$$
\begin{aligned}
\sum_{i=0}^{m}\left(c_{i}^{q}-c_{i}^{q-1}\right) L_{i}^{a}\left(x_{p}\right)= & \Delta t \cdot \sum_{j=1}^{r} h_{j}\left(x_{p}, t_{q}\right) \sum_{i=n_{j}}^{m} \sum_{k=n_{j}}^{i} c_{i}\left(t_{q}\right) A_{i, k}^{\alpha_{j}} x_{p}^{k-\alpha_{j}\left(x_{p}, t_{q}\right)} \\
& +\Delta t \cdot N\left(\sum_{i=0}^{m} c_{i}\left(t_{q}\right) L_{i}^{a}\left(x_{p}\right)\right), q=1,2, \ldots, N .
\end{aligned}
$$

Combining equations (3.4) and (3.5), we have a system of non-linear equations as follows

$$
\mathbf{F}=\mathbf{0},
$$

where $\mathbf{0}$ is zero vector with $m+1$ components and $\mathbf{F}$ is a vector whose components are given by

$$
\begin{aligned}
\mathbf{F}_{p}= & \sum_{i=0}^{m}\left(c_{i}^{q}-c_{i}^{q-1}\right) L_{i}^{a}\left(x_{p}\right)=\Delta t \cdot \sum_{j=1}^{r} h_{j}\left(x_{p}, t_{q}\right) \sum_{i=n_{j}}^{m} \sum_{k=n_{j}}^{i} c_{i}\left(t_{q}\right) A_{i, k}^{\alpha_{j}} x_{p}^{k-\alpha_{j}\left(x_{p}, t_{q}\right)} \\
& +\Delta t \cdot N\left(\sum_{i=0}^{m} c_{i}\left(t_{q}\right) L_{i}^{a}\left(x_{p}\right)\right), p=1,2, \ldots, m-n_{r}+1 \\
\mathbf{F}_{m-n_{r}+2}= & \sum_{i=0}^{m} c_{i}\left(t_{q}\right) L_{i}^{a}\left(b_{0}\right)-d_{0}\left(t_{q}\right), \\
\mathbf{F}_{m-n_{r}+3}= & \sum_{i=0}^{m} c_{i}\left(t_{q}\right) L_{i}^{a}\left(b_{1}\right)-d_{1}\left(t_{q}\right), \\
\ldots & \\
\mathbf{F}_{m+1}= & \sum_{i=0}^{m} c_{i}\left(t_{q}\right) L_{i}^{a}\left(b_{n_{r}-1}\right)-d_{n_{r}-1}\left(t_{q}\right) .
\end{aligned}
$$

For the non-linear system (3.6) with unknown $V^{q}(q=1, \ldots, N)$, the following iteration formula can be written by using Newton iteration method

$$
\begin{aligned}
V^{q, k+1} & =V^{q, k}-J^{-1}\left(V^{q, k}\right) . \mathbf{F}\left(V^{q, k}\right), \\
V^{q, 0} & =V^{q-1}, q=1, \ldots, N, k=0,1, \ldots
\end{aligned}
$$


where $J^{-1}\left(V^{q, k}\right)$ is the inverse of the Jacobian matrix, $V^{q, k}$ is approximate solution of $V^{q}$. Using iteration formula (3.9), approximate solution of the problem (1.1)-(1.3) is obtained.

\section{Applications}

Example 1. Consider nonlinear space fractional partial differential equation with initial and boundary conditions

$$
\begin{aligned}
& u_{t}(x, t)={ }^{\alpha(x, t)} \Gamma(5-\alpha(x, t)) \cdot{ }^{C} D_{x}^{\alpha(x, t)} u(x, t)-x^{\beta(x, t)} \Gamma(5-\beta(x, t)) \\
&{ }^{C} D_{x}^{\beta(x, t)} u(x, t)+u^{3}(x, t)-\left(x^{4}-x^{2}+1\right) \sin (t) u^{2}(x, t)+\cos (t)\left(x^{4}-x^{2}+1\right) \\
&-\frac{\Gamma(5-\beta(x, t))}{\Gamma(3-\beta(x, t))} 2 x^{2} \sin (t), 0<x<2,0<t \leq 1 \\
& u(x, 0)= 0,0<x<2 \\
& u(0, t)= \sin (t), u(1, t)=\sin (t), u(2, t)=13 \sin (t), 0<t \leq 1 \\
& \text { where } \alpha(x, t)=\frac{6+\sin (x+t)}{3}, \beta(x, t)=\frac{4+\sin (x+t)}{3} .
\end{aligned}
$$

The exact solution is $u_{e}(x, t)=\sin (t)\left(x^{4}-x^{2}+1\right)$. Assume that the solution of the problem (4.1)-(4.3) can be written as follows

$$
u_{4}(x, t)=\sum_{i=0}^{4} c_{i}(t) L_{i}^{0}(x) .
$$

Substituting equation (4.4) into the boundary conditions (4.3) at the points $t_{q}$, we have

$$
\begin{aligned}
& \mathbf{F}_{3}=c_{0}\left(t_{q}\right)+c_{1}\left(t_{q}\right)+c_{2}\left(t_{q}\right)+c_{3}\left(t_{q}\right)+c_{4}\left(t_{q}\right)-\sin \left(t_{q}\right), \\
& \mathbf{F}_{4}=c_{0}\left(t_{q}\right)-1 / 2 c_{2}\left(t_{q}\right)-2 / 3 c_{3}\left(t_{q}\right)-5 / 8 c_{4}\left(t_{q}\right)-\sin \left(t_{q}\right), \\
& \mathbf{F}_{5}=c_{0}\left(t_{q}\right)-c_{1}\left(t_{q}\right)-c_{2}\left(t_{q}\right)-1 / 3 c_{3}\left(t_{q}\right)+1 / 3 c_{4}\left(t_{q}\right)-13 \sin \left(t_{q}\right) .
\end{aligned}
$$

For $m=4$, collocation points are taken as $x_{1}=0.25, x_{2}=0.75$. $\mathbf{F}_{1}$ and $\mathbf{F}_{2}$ can be written from (3.7) for the points $x_{1}=0.25, x_{2}=0.75$. By using the iteration formula (3.9) with the initial data $V^{0}=(0,0,0,0,0)^{*}$, the absolute errors for the approximate solution $u_{4}(x, t)$ are computed for $N=10^{3} ; 10^{4} ; 10^{5} ; 10^{6} ; 10^{7}$ at $t=0.25 ; 0.75$. Table 1 shows that the absolute error goes to the zero as the value $N$ increases. Figure 1 shows 3D plot of the absolute error for the approximate solution $u_{4}(x, t)$ for $N=10^{6}$, $0<x<2$ and $0<t<1$.

Example 2. Consider nonlinear space fractional partial differential equation with initial and boundary conditions

$$
\begin{aligned}
u_{t}(x, t)= & x^{\alpha(x, t)} \Gamma(6-\alpha(x, t)) \cdot{ }^{C} D_{x}^{\alpha(x, t)} u(x, t)-x^{\beta(x, t)} \Gamma(6-\beta(x, t)) \cdot{ }^{C} D_{x}^{\beta(x, t)} u(x, t) \\
& +\left(x^{5}-2\right)\left(t^{2}-t\right) u(x, t)-u^{2}(x, t)+\left(x^{5}-2\right)(2 t-1), 0<x<5,0<t \leq 1,
\end{aligned}
$$


TABLE 1 . The absolute errors for $u_{4}(x, t), N=10^{3} ; 10^{4} ; 10^{5} ; 10^{6}$; $10^{7}$ at $t=0.25 ; 0.75$ in Example 1 .

\begin{tabular}{|c|c|c|c|c|c|c|c|c|c|c|}
\hline & \multicolumn{2}{|c|}{$N=10^{3}$} & \multicolumn{2}{|c|}{$N=10^{4}$} & \multicolumn{2}{|c|}{$N=10^{5}$} & \multicolumn{2}{|c|}{$N=10^{6}$} & \multicolumn{2}{|c|}{$N=10^{7}$} \\
\hline & \multicolumn{2}{|c|}{$0.25 t=0.75$} & & & & \\
\hline & \multirow{2}{*}{\begin{tabular}{|l|l|l}
2 & $10^{-5}$ \\
4 & $210^{-5}$
\end{tabular}} & $\begin{array}{r}5 t=0.75 \\
6.10^{-5}\end{array}$ & \multicolumn{8}{|c|}{ 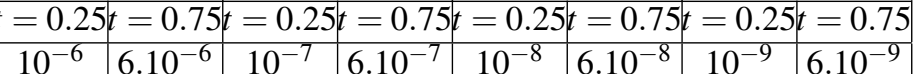 } \\
\hline \multirow{2}{*}{\multicolumn{2}{|c|}{$\begin{array}{l}2.10^{-5} \\
2.10^{-5}\end{array}$}} & & & & & & & & & \\
\hline & & & & & & & & & & \\
\hline \multirow{2}{*}{\multicolumn{2}{|c|}{$\begin{array}{l}2.10^{-5} \\
22.10^{-5}\end{array}$}} & & & & & & & & & \\
\hline & & & & & & & & & & \\
\hline \multicolumn{2}{|c|}{$1.44 .10^{-5}$} & & & & & & 4.1 & & & \\
\hline \multirow{2}{*}{\multicolumn{2}{|c|}{\begin{tabular}{|l|l}
$5.10^{-5}$ \\
$8.10^{-5}$
\end{tabular}}} & & & & & & & & & \\
\hline & & 10 & 4.10 & & 4.10 & 3.1 & 4.10 & 3.1 & 4.10 & 10 \\
\hline
\end{tabular}

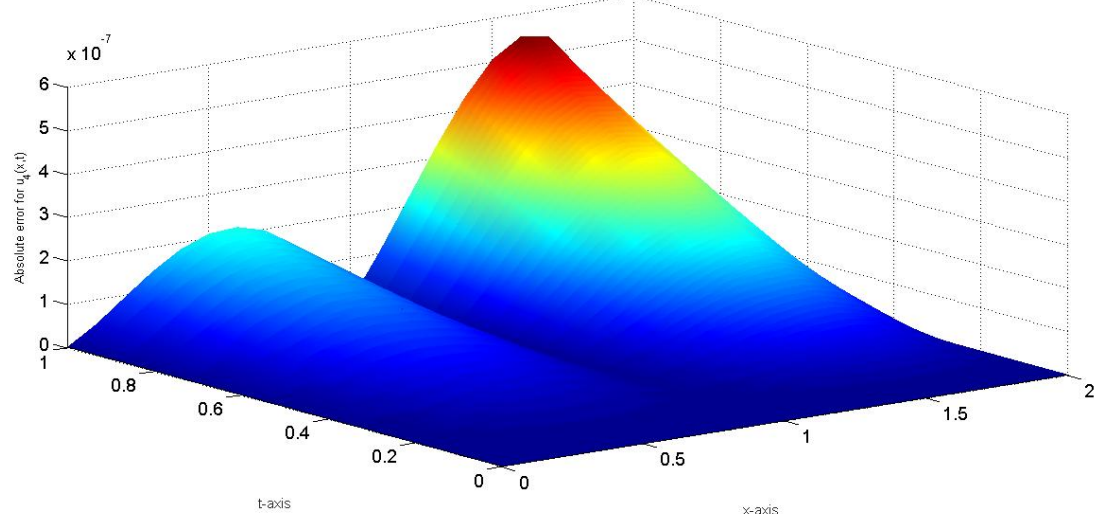

FIGURE 1. The behavior of the absolute error for the approximate solution $u_{4}(x, t)$ for $N=10^{6}, 0<x<2$ and $0<t<1$ in Example 1 .

$u(x, 0)=0,0<x<5, \quad u(0, t)=2 t-2 t^{2}, u(5, t)=3123 t^{2}-3123 t, 0<t \leq 1$,

where $\alpha(x, t)=\frac{5+\sin (x+t)}{3}, \beta(x, t)=\frac{1+\sin (x+t)}{4}$.

The exact solution is $u_{e}(x, t)=\left(t^{2}-t\right)\left(x^{5}-2\right)$. By using the iteration formula (3.9) for $m=5, a=0$ with the initial data $V^{0}=(0,0,0,0,0,0)^{*}$, the absolute errors for the approximate solution $u_{5}(x, t)$ are computed for $N=10^{4} ; 10^{5} ; 10^{6} ; 10^{7}$ at $t=0.5$. The influence of the value $N$ on the absolute error is presented in Table 2. Table 2 shows that the value of the absolute error goes to zero as the value $N$ increases. Figure 2 shows 3D plot of the approximate solution $u_{5}(x, t)$ for $N=10^{4}, 3<x<5$ and $0<t<1$. 
TABLE 2. The absolute errors for $u_{5}(x, 0.5)$ in Example 2.

\begin{tabular}{|c|c|c|c|c|}
\hline$x$ & $N=10^{4}$ & $N=10^{5}$ & $N=10^{6}$ & $N=10^{7}$ \\
\hline 0.4 & $3.10^{-6}$ & $3.10^{-7}$ & $4.10^{-8}$ & $4.10^{-9}$ \\
\hline 1.2 & $10^{-4}$ & $10^{-5}$ & $10^{-6}$ & $10^{-7}$ \\
\hline 2 & $10^{-3}$ & $10^{-4}$ & $10^{-5}$ & $10^{-6}$ \\
\hline 2.4 & $2.10^{-3}$ & $2.10^{-4}$ & $2.10^{-5}$ & $2.10^{-6}$ \\
\hline 3.2 & $5.10^{-3}$ & $5.10^{-4}$ & $5.10^{-5}$ & $5.10^{-6}$ \\
\hline 3.6 & $7.10^{-3}$ & $7.10^{-4}$ & $7.10^{-5}$ & $7.10^{-6}$ \\
\hline 4 & $7.10^{-3}$ & $7.10^{-4}$ & $7.10^{-5}$ & $7.10^{-6}$ \\
\hline 4.4 & $7.10^{-3}$ & $7.10^{-4}$ & $7.10^{-5}$ & $7.10^{-6}$ \\
\hline 4.8 & $3.10^{-3}$ & $3.10^{-4}$ & $3.10^{-5}$ & $3.10^{-6}$ \\
\hline
\end{tabular}

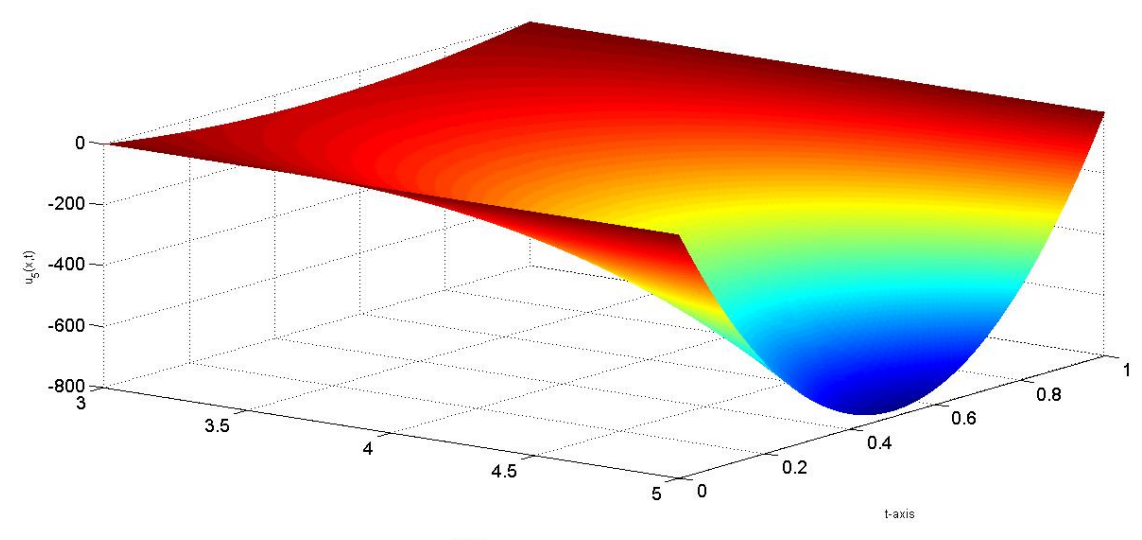

FIGURE 2. The behavior of the approximate solution $u_{5}(x, t)$ for $N=10^{4}, 3<x<5$ and $0<t<1$ in Example 2 .

Example 3. Let us consider the following nonlinear space fractional partial differential equation with initial and boundary conditions

$$
\begin{aligned}
u_{t}(x, t)= & { }^{C} D_{x}^{\alpha(x, t)} u(x, t)+u^{2}(x, t)-\exp (x) t^{2} u(x, t) \\
& +2 t \exp (x)-t^{2} x^{2-\alpha(x, t)} E_{1,3-\alpha(x, t)}(x), 0<x<1,0<t<1, \\
u(x, 0)= & 0,0<x<1, \quad u(0, t)=t^{2}, u(1, t)=\exp (1) t^{2}, 0<t \leq 1,
\end{aligned}
$$

where $\alpha(x, t)=\frac{4+\sin (x+t)}{3}$ and $E_{1,3-\alpha(x, t)}(x)$ is the Mittag-Leffler function (see, for example, [17]). Note that the exact solution to this problem is $u_{e}(x, t)=\exp (x) t^{2}$. By using the iteration formula (3.9) with zero initial condition, we obtain approximate solution of the problem for $m=3, a=0 ; 4 ; 5$. In Table 3 , the absolute errors 
between the exact solution $u_{e}(x, t)$ and the approximate solution $u_{m}(x, t), m=3,4,5$, at $t=0.5$ for the values $N=10^{6}$ are given. Table 3 shows how the change of the values $m$ effects the obtained results. As shown in Table 3 , as the values $m$ increase, the approximate solution approaches to the exact solution. Figure 3 presents $3 \mathrm{D}$ plot of the absolute error for the approximate solution $u_{5}(x, t)$ for $N=10^{5}, 0<x<1$ and $0<t<1$.

TABLE 3. The absolute errors for $u_{m}(x, t), m=3 ; 4 ; 5 ; 10^{6}$ at $t=$ $0.2 ; 0.8$ in Example 3.

\begin{tabular}{|c|c|c|c|c|c|c|}
\hline$x$ & \multicolumn{2}{|c|}{$m=3$} & \multicolumn{2}{c|}{$m=4$} & \multicolumn{2}{c|}{$m=5$} \\
\cline { 2 - 7 } & $t=0.2$ & $t=0.8$ & $t=0.2$ & $t=0.8$ & $t=0.2$ & $t=0.8$ \\
\hline 0.2 & $3.10^{-5}$ & $6.10^{-4}$ & $2.10^{-6}$ & $3.10^{-5}$ & $7.10^{-8}$ & $7.10^{-7}$ \\
\hline 0.4 & $6.10^{-5}$ & $10^{-3}$ & $10^{-6}$ & $2.10^{-6}$ & $3.10^{-7}$ & $2.10^{-6}$ \\
\hline 0.6 & $4.10^{-5}$ & $5.10^{-4}$ & $2.10^{-6}$ & $5.10^{-5}$ & $2.10^{-7}$ & $6.10^{-8}$ \\
\hline 0.8 & $3.10^{-5}$ & $6.10^{-4}$ & $2.10^{-7}$ & $10^{-5}$ & $5.10^{-8}$ & $2.10^{-6}$ \\
\hline
\end{tabular}

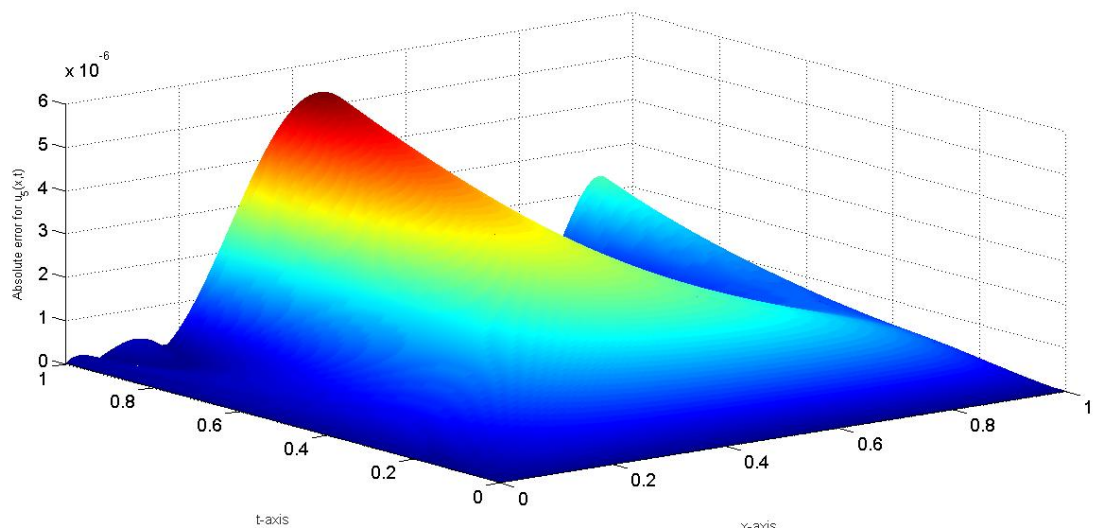

FIGURE 3. The behavior of the absolute value of the approximate solution $u_{5}(x, t)$ for $N=10^{5}, 0<x<1$ and $0<t<1$ in Example 3 .

Example 4. Let us consider the following nonlinear space fractional partial differential equation with initial and boundary conditions

$$
\begin{aligned}
u_{t}(x, t)= & \exp (-t) x \cdot{ }^{C} D_{x}^{\alpha(x, t)} u(x, t)+u^{3}(x, t)-\exp (t) \cos (t)\left(x^{3}-x^{2}\right) u^{2}(x, t) \\
& +u(x, t)+\cos (t) x^{3-\alpha(x, t)} \frac{2}{\Gamma(3-\alpha(x, t))}-\cos (t) x^{4-\alpha(x, t)} \frac{6}{\Gamma(4-\alpha(x, t))}
\end{aligned}
$$




$$
\begin{gathered}
-\exp (t) \sin (t)\left(x^{3}-x^{2}\right), 0<x<1,0<t<1, \\
u(x, 0)=x^{3}-x^{2}, 0<x<1, \quad u(0, t)=0, u(1, t)=0,0<t \leq 1,
\end{gathered}
$$

where $\alpha(x, t)=\frac{5+\sin (x t)}{4}$. Note that the exact solution to this problem is $u_{e}(x, t)=$ $\exp (t) \cos (t)\left(x^{3}-x^{2}\right)$. By using the iteration formula (3.9) for $m=3, a=0$ with the initial data $V^{0}=(4,-14,16,-6)^{*}$, the absolute errors for the approximate solution $u_{3}(x, t)$ are computed for $N=10^{3} ; 10^{4} ; 10^{5} ; 10^{6}$ at $t=0.2 ; 0.9$. The influence of the value $N$ on the absolute error is presented in Table 4. Table 4 shows that the value of the absolute error goes to zero as the value $N$ increases. Figure 4 shows 3D plot of the approximate solution $u_{3}(x, t)$ for $N=10^{4}, 0<x<1$ and $0<t<1$.

TABLE 4 . The absolute errors for $u_{3}(x, t), N=10^{3} ; 10^{4} ; 10^{5} ; 10^{6}$ at $t=0.2 ; 0.9$ in Example 4.

\begin{tabular}{|c|c|c|c|c|c|c|c|c|}
\hline \multirow{3}{*}{$x$} & \multicolumn{2}{|c|}{$N=10^{3}$} & \multicolumn{2}{c|}{$N=10^{4}$} & \multicolumn{2}{c|}{$N=10^{5}$} & \multicolumn{2}{c|}{$N=10^{6}$} \\
\cline { 2 - 9 } & $t=0.2$ & $t=0.9$ & $t=0.2$ & $t=0.9$ & $t=0.2$ & $t=0.9$ & $t=0.2$ & $t=0.9$ \\
\hline 0 & $9.10^{-16}$ & $2.10^{-15}$ & $9.10^{-16}$ & $4.10^{-15}$ & $3.10^{-15}$ & 0 & $9.10^{-16}$ & $4.10^{-15}$ \\
\hline 0.2 & $9.10^{-7}$ & $4.10^{-5}$ & $9.10^{-8}$ & $4.10^{-6}$ & $9.10^{-9}$ & $3.10^{-7}$ & $9.10^{-10}$ & $4.10^{-8}$ \\
\hline 0.4 & $2.10^{-6}$ & $7.10^{-5}$ & $2.10^{-7}$ & $7.10^{-6}$ & $2.10^{-8}$ & $6.10^{-7}$ & $2.10^{-9}$ & $7.10^{-8}$ \\
\hline 0.6 & $3.10^{-6}$ & $8.10^{-5}$ & $3.10^{-7}$ & $8.10^{-6}$ & $3.10^{-8}$ & $8.10^{-7}$ & $3.10^{-9}$ & $8.10^{-8}$ \\
\hline 0.8 & $3.10^{-6}$ & $6.10^{-5}$ & $3.10^{-7}$ & $6.10^{-6}$ & $3.10^{-8}$ & $6.10^{-7}$ & $3.10^{-9}$ & $6.10^{-8}$ \\
\hline 1 & $9.10^{-16}$ & 0 & $9.10^{-16}$ & $9.10^{-16}$ & $9.10^{-16}$ & 0 & 0 & $2.10^{-15}$ \\
\hline
\end{tabular}

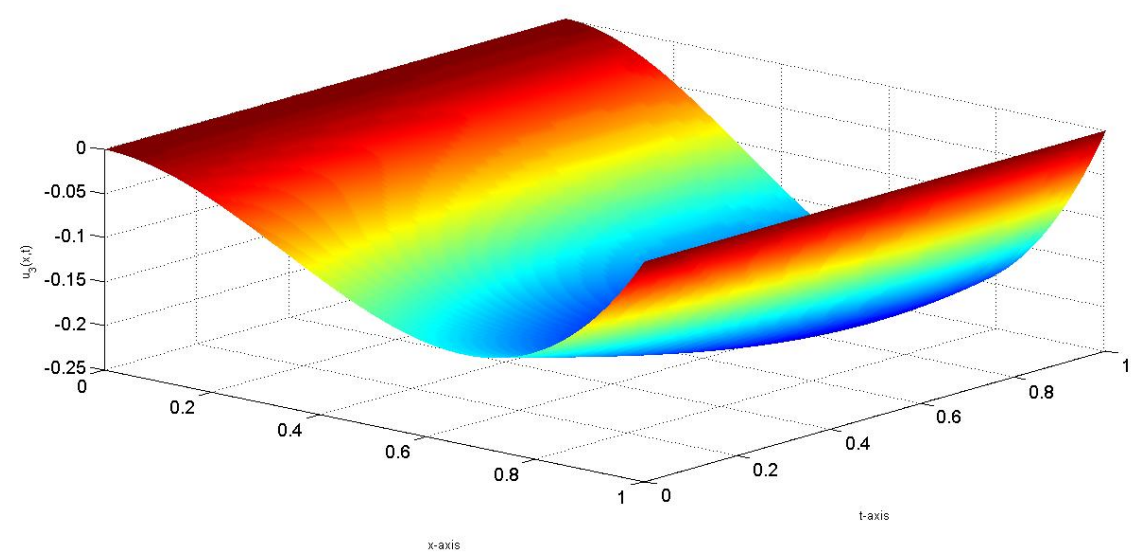

FIGURE 4. The behavior of the approximate solution $u_{3}(x, t)$ for $N=10^{4}, 0<x<1$ and $0<t<1$ in Example 4 . 


\section{CONCLUSiON}

In this paper, numerical solutions of the multi-term variable-order space fractional nonlinear partial differential equations with initial and boundary conditions have been obtained. The space variable order fractional derivative is defined in the Caputo sense. Spectral Laguerre collocation method and finite difference method have been applied to the problem and the problem has been transformed into an algebraic system of the nonlinear equations. The nonlinear system has been solved by using Newton iteration. Some numerical examples have been given to clarify the validity and accuracy of the proposed technique. The obtained numerical results are compared with exact solutions. The proposed scheme leads to results that are in excellent agreement with the exact solutions and show that the algorithm is efficient. All numerical computations were performed in MATLAB.

\section{REFERENCES}

[1] M. Abbas and M. Ragusa, "Solvability of Langevin equations with two Hadamard fractional derivatives via Mittag-Leffler functions," arXiv:2006.07608, pp. 1-17, 2020.

[2] R. Bagley and P. Torvik, "On the appearance of the fractional derivative in the behavior of real materials," J. Appl. Mech., vol. 51, no. 2, pp. 294-298, 1984, doi: 10.1115/1.3167615.

[3] S. Benbernou, S. Gala, and M. Ragusa, "On the regularity criteria for the 3D magnetohydrodynamic equations via two components in terms of BMO space," Math. Method Appl. Sci., vol. 37, no. 15, pp. 2320-2325, 2014, doi: 10.1002/mma.2981.

[4] A. Borhanifar, M. Ragusa, and S. Valizadehaz, "High-order numerical method for twodimensional Riesz space fractional advection-dispersion equation," arXiv:2006.04111, pp. 1-18, 2020.

[5] Y. Chen, L. Liu, B. Li, and Y. Sun, "Numerical solution for the variable order linear cable equation with Bernstein polynomials," Appl.Math.Comput., vol. 238, no. 1, pp. 329-341, 2014, doi: 10.1016/j.amc.2014.03.066.

[6] C. Coimbra, "Mechanics with variable-order differential operators," Ann. Phys., vol. 12, pp. 692703, 2003, doi: 10.1002/andp.200310032.

[7] H. Dehestani and Y. Ordokhani, "An efficient collocation method for solving the variable-order time-fractional partial differential equations arising from the physical phenomenon," Int. J. Appl. Math. Comput. Sci. , vol. 12, no. 12, pp. 241-252, 2018, doi: 10.5281/zenodo. 2022075.

[8] H. Hassani and E. Naraghirad, " A new computational method based on optimization scheme for solving variable-order time fractional Burgers' equation,” Math. Comput. Simulat., vol. 162, pp. 1-17, 2019, doi: 10.1016/j.matcom.2019.01.002.

[9] H. Hassani, J. Tenreiro Machado, Z. Avazzadeh, and E. Naraghirad , "Generalized shifted Chebyshev polynomials: Solving a general class of nonlinear variable order fractional PDE," Commun. Nonlinear Sci. Numer. Simulat. , vol. 85, p. 105229, 2020, doi: 10.1016/j.cnsns.2020.105229.

[10] M. H. Heydari, Z. Avazzadeh, and Y. Yang, " A computational method for solving variable-order fractional nonlinear diffusion-wave equation," Appl. Math. Comput., vol. 352, pp. 235-248, 2019, doi: 10.1016/j.amc.2019.01.075.

[11] M. Hosseininia, M. Heydari, F. M. Ghaini, and Z. Avazzadeh, " A wavelet method to solve nonlinear variable-order time fractional 2D Klein-Gordon equation,” Comput. Math. Appl. , vol. 78, no. 12, pp. 3713-3730, 2019, doi: 10.1016/j.camwa.2019.06.008. 
[12] S. Irandoust-Pakchin, S. Abdi-Mazraeh, and A. Khani, "Numerical solution for a variable-order fractional nonlinear cable equation via Chebyshev cardinal functions," Comput. Math. Math. Phys., vol. 57, pp. 2047-2056, 2017, doi: 10.1134/S0965542517120120.

[13] M. M. Khader and M. M. Babatin, "On approximate solutions for fractional logistic differential equation,” Math. Probl. Eng., vol. 2013, pp. 1-7, 2013, doi: 10.1155/2013/391901.

[14] F. Mainardi, Fractional calculus: 'Some basic problems in continuum and statistical mechanics', In: A. Carpinteri, F. Mainardi, Editors, Fractals and Fractional Calculus in Continuum Mechanics. New York: Springer-Verlag, 1997.

[15] B. Moghaddam and J. Machado, "A stable three-level explicit spline finite difference scheme for a class of nonlinear time variable order fractional partial differential equations," Comput. Math. Appl., vol. 73, no. 6, pp. 1262-1269, 2017, doi: 10.1016/j.camwa.2016.07.010.

[16] F. Mohammadi and H. Hassani, "Numerical solution of two-dimensional variable-order fractional optimal control problem by generalized polynomial basis," J. Optim. Theory Appl. , vol. 180, pp. 536-555, 2019, doi: 10.1007/s10957-018-1389-z.

[17] I. Podlubny, Fractional Differential Equations. San Diego: Academic Press, 1999.

[18] I. Podlubny, "Geometric and physical interpretation of fractional integration and fractional differentiation,” Fract. Calculus. App. Anal., vol. 5, no. 4, pp. 367-386, 2002.

[19] M. Ragusa and A. Razani, "Weak solutions for a system of quasilinear elliptic equations," Contrib. Math., vol. 1, pp. 11-16, 2020, doi: 10.47443/cm.2020.0008.

[20] M. Ragusa and A. Scapellato, "Mixed Morrey spaces and their applications to partial differential equations," Nonlinear Anal. Theory Methods Appl., vol. 151, pp. 51-65, 2017, doi: 10.1016/j.na.2016.11.017.

[21] L. Ramirez, C. Coimbra, and M. Kobayashi, "Variable order constitutive relation for viscoelasticity," Ann. Phys., vol. 16, pp. 543-552, 2007, doi: 10.1002/andp.200710246.

[22] Y. Shekari, A. Tayebi, and M. H. Heydari, " A meshfree approach for solving 2D variable-order fractional nonlinear diffusion-wave equation," Comput. Methods Appl. Mech. Engrg., vol. 350, no. 15, pp. 154-168, 2019, doi: 10.1016/j.cma.2019.02.035.

[23] S. Shen, F. Liu, J. Chen, I. Turner, and V. Anh, " Numerical techniques for the variable order time fractional diffusion equation,” Appl.Math.Comput., vol. 218, no. 22, pp. 10861-10 870, 2012, doi: 10.1016/j.amc.2012.04.047.

[24] H. Sun, Y. Chen, and W. Chen, "Random-order fractional differential equation models," Sign. Process., vol. 91, pp. 525-530, 2011, doi: 10.1016/j.sigpro.2010.01.027.

[25] N. H. Sweilam, S. M. Al-Mekhlafi, and A. O. Albalawi, “ A novel variable-order fractional nonlinear Klein Gordon model: A numerical approach," Numer. Methods Partial Differential Eq. , vol. 35, no. 5, pp. 1617-1629, 2019, doi: 10.1002/num.22367.

[26] J. Wang, A. Ibrahim, and D. O'Regan, "Finite approximate controllability of Hilfer fractional semilinear differential equations," Miskolc Math. Notes, vol. 21, no. 1, pp. 489-507, 2020, doi: 10.18514/MMN.2020.2921.

[27] W. Zahra and M. Hikal, " Non standard finite difference method for solving variable order fractional optimal control problems," J. Vib. Control, vol. 23, no. 6, pp. 948-958, 2017, doi: $10.1177 / 1077546315586646$.

\section{Author's address}

\section{H. Çerdik Yaslan}

Pamukkale University, Department of Mathematics, Denizli, Turkey

E-mail address: hcerdikepau.edu.tr 